\title{
What Is the Value of Obesity Research?
}

\author{
John E. Blundell Johannes Hebebrand ${ }^{\mathrm{b}}$ Jean-Michel Oppert, on behalf of the EASO Scientific \\ Advisory Board expert group
}

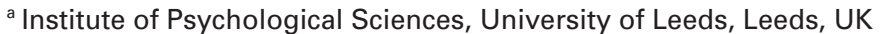

${ }^{b}$ Department of Child and Adolescent Psychiatry, University of Duisburg-Essen, Essen, Germany

${ }^{c}$ Department of Nutrition, Pitei-Salpetriere Hospital (AP-HP), Paris, France

The European Association for the Study of Obesity (EASO) was founded in 1986 with the aims 'to promote research into obesity, facilitate contact between individuals and organisations, and promote action that tackles the epidemic of obesity'. After two and half decades of existence, EASO has substantially expanded its activities and at the time of writing, EASO comprises national obesity associations in 40 member states (see www.easoobesity.org). In parallel, the potential for obesity research in Europe has significantly increased, as shown by past and ongoing large-scale EU-funded research projects in the field of obesity and related topics.

In order to better understand this extensive and culturally diverse community of partners, EASO wished to get a better understanding of ongoing activities related to obesity research and management of research in Europe. One immediate need was to be informed about the need for research in various regions, the capacity of the European research community, and the impact of research on the social, psychological, economic and medical issues associated with the obesity epidemic. The initial step was to convene a meeting of experts to provide a snap-shot review of the current status and effectiveness of obesity research in Europe. This group was termed EASO Scientific Advisory Board (SAB) and was chaired by John Blundell. The experts were given a series of questions to direct their thinking towards specific problems and issues. The SAB met for a 1-day meeting on January 16, 2010 at the Department of Nutrition, Pitié-Salpêtrière Hospital in Paris under the patronage of Jean-Michel Oppert, the President of EASO. In order to furnish the SAB with a full picture of the landscape of research, questionnaires were sent to the presidents of every national obesity association in each member state $(n=40)$, and also to the chairpersons of each of the EASO Task Forces (TF): Childhood Obesity TF - Ram Weiss; Obesity Management TF - Constantine Tsigos; Public
Health and Prevention TF -Tommy Visscher. The responses to these questionnaires helped to guide the discussion of the SAB.

The invited members of the SAB were: Carlos Dieguez (Spain), Johannes Hebebrand (Germany), Dominique Langin (France), Nick Finer (UK), Jean-Michel Oppert (France), Thorkild I.A. Sørensen (Denmark), and Jane Wardle (UK). These members represented a wide cross-section of research in obesity, ranging from adipose tissue biology to neuroscience through genetics, behavioural sciences, epidemiology and public health as well as clinical management of obesity, although the coverage was not exhaustive. This brief synopsis is to provide an overview of the major issues discussed within the SAB.

Although it is known that approximately $1-5 \%$ of obese children and $1 \%$ of obese adults harbour mutations in the melanocortin-4 receptor gene $(M C 4 R)$, it is assumed that 'normal' obesity is a polygenic condition. The advent of genome wide association studies (GWAS) and the use of large samples for the first time allowed the large-scale detection and validation of gene variants, thus representing a major step forward. However, despite a substantial investment (financial and intellectual) into the genetics of obesity, the results are disappointing in that only a small fraction of the variance of the BMI $\left(\mathrm{kg} / \mathrm{m}^{2}\right)$ can be explained. Meta-analyses of GWAS have revealed that approximately 30 obesity polygenes influence BMI; the effect sizes of these polygenes are (very) small. The functionally relevant variation at the DNA level needs to be determined for all of the respective association signals. The notion that the CNS is important in the development of obesity is supported by the fact that most of the respective genes are expressed centrally. The heritability of obesity is estimated between 40 and $70 \%$, but the currently known polygenes do not provide a basis for the prediction of who will

\section{KARGER}

Fax +49 761452071

Information@Karger.de

www.karger.com
(ㄷ) 2010 S. Karger GmbH, Freiburg

Accessible online at:

www.karger.com/ofa
Prof. Dr. John E. Blundell

Institute of Psychological Sciences

University of Leeds

Leeds LS2 9JT, UK

J.E.Blundell@leeds.ac.uk 
become obese. Prediction is substantially better if based on an evaluation of the weight status of parents and siblings. Ten years ago confidence was high that genetics would provide insights into the causes, mechanisms, and solutions for obesity. In the light of the current outcomes, the SAB discussed the future for obesity genetics. Do we need an ongoing debate concerning the direction of genetic research and the financial investment to be committed? What is the part of epigenetics and other 'omics' in this field? Should EASO have a role in that debate?

In the closely related area of adipose tissue biochemistry, the key areas of interest are: how does adipose tissue develop (adipogenesis) and how does it lead to pathology? There appears to be a mismatch between the animal (mainly rodents) and human research since usually in animals adipose tissue is studied during growth (on a high-fat diet) and in humans after growth (once a person has become obese). This may be logistically inevitable but it questions whether or not animal research is truly throwing light on human processes. Specifically, it can be asked: what are the implications for humans of studies on inflammation in animals? The situation is clearly complex, not least because of the different cell types in adipose tissue and the extent to which each of these cell types plays a role in leading to impairment in muscle or liver.

However, there appears to be a revival of interest in brown adipose tissue (BAT) following little interest over 15 years. Why should this be? Interest in BAT declined after it transpired that the $\beta 3$ receptor was functional in mouse, but less so in human adipose tissue. It remains to be seen how the scientific community will react to - and absorb - the new findings on BAT. At this stage in the progress of research, we can ask: how does adipose tissue research really inform us about the aetiology of obesity, and how can research outcomes be translated into useful clinical practice? Are there good examples of research in this area helping to understand the medical complications produced as a result of accumulating fat in the body?

Similar questions can be asked of the relationship between neuroscience research and obesity. The neural networks and matrices of receptor interactions have been quite well constructed within the framework of 'energy homeostasis'. Considerable advances have been made in the last 15 years stimulated by the discovery of leptin, and, within the hypothalamus, neuropeptide Y (NPY), agouti-related peptide (AgRP), melanocyte-stimulating hormones $(\mathrm{MCH})$, Pro-opiomelanocortin (POMC) and orexins provide a basis for describing pathways for weight gain and loss. The link between peripheral peptides such as ghrelin, Peptide YY (PYY), glucagonlike peptide-1 (GLP-1) and receptors in the brain has further articulated the mechanisms underlying the control of energy intake. Opioids and the endocannabinoids are usually subscribed under a related hedonic system. These systems, together with the previously known biogenic amines provided a neural explanation for the mechanism of action of antiobesity drugs such as sibutramine (re-uptake inhibitor of serotonin
(5-HT) and norepinephrine (NE)) and rimonabant and taranabant (CB1-R inverse agonists), and for the possible development of future drugs based on $\mathrm{Y} 2$ and $\mathrm{Y} 4$ receptors, $\mathrm{MCH}$ antagonists and others. However, the failure of CNS drugs to produce a favourable risk-benefit ratio has lead to the prohibition of the CB1-R inverse agonists and the removal of approval for sibutramine in Europe (and possibly in the USA in the near future). The problem is that the weight loss achieved by most drugs is in the order of $3.5-6.0 \mathrm{~kg} /$ year - which is very modest. When set against an increased risk of cardiovascular and psychiatric adverse events, the advantages seem trivial, although there may well be some additional beneficial effects with some of these drugs independently of the weight loss (e.g. improvement in lipid profile, insulin resistance, etc).

Currently, there is considerable interest in developing pharmacotherapy using combinations of drugs, including topirimate/phentermine, naltrexone/bupropion, naltrexone/ zonisimide, amylin/metroleptin and others. The extent to which these drugs can produce a more satisfactory risk-benefit ratio remains to be demonstrated; they certainly have the potential to produce a greater weight loss. At the present time neither the Food and Drug Administration (FDA) nor the European Medicines Agency (EMEA) have yet issued an approval for any of these combinations. Whilst it can be claimed that CNS research has contributed a theoretical rationale for the development of drugs that have appeared in the last decade, the question still remains whether or not brain research can provide a therapeutic solution to the problem of obesity.

One technological advance that should be mentioned is the use of functional magnetic resonance imaging (fMRI) scanning to throw light on brain mechanisms responsible for appetite control or weight regulation. This issue is currently generating widespread interest. However, although the procedure can illuminate some of the hedonic and homeostatic controls over eating behaviour, the resolving power is not very high and fMRI may be a tool that allows description rather than explanation. Nevertheless, this procedure is sure to be implicated in the development of novel classes of anti-obesity drugs.

The issue of pharmacological treatment draws attention to the success of other management procedures. At present time the use of behavioural and nutritional strategies and targeted lifestyle strategies offer a degree of weight loss approximately double that provided by drugs. Neither approach meets the expectations of most patients, and both rely on degrees of patient compliance that are rarely maintained. What does research tells us about ways to strengthen compliance or to prevent non-compliance? Moreover, there is a question of when to intervene? In the early phase of weight gain (an overweight period), or only after frank obesity has developed? This raises the key issue of prevention versus treatment and poses questions for the tactics of population management of obesity from a public health perspective. Is this a question that can be solved by research or rather by socio-economic analysis? 
In this discussion, the role of bariatric surgery acquires considerable prominence. The success of the procedures (particularly gastric bypass) in achieving weight loss is beyond dispute; however there are risks, including death. It is paradoxical that surgical procedures can be carried out without a preceding clinical trial and without, apparently, the need for consideration by a statuary (or licensing) body of the possibility of risks. From a scientific perspective, bariatric procedures are fascinating since they are beginning to throw further light on the role of gastrointestinal peptides in the therapeutic response, and therefore (although less convincingly) in the overall control of homeostasis. What is the future of bariatric surgery? Are there still more sophisticated procedures yet to be developed? And what percentage of the obese community should be treated through surgery?

One major question for EASO is how to evaluate the effectiveness (economic, social, psychological and medical) of the various modalities of managing obesity, and where research investment should be focussed.

Considering the role of physical activity for obesity, the current position is that increasing physical activity is essential for the prevention of weight gain and that it makes a measurable contribution to weight loss. However, it should be noted that the scientific community is quite divided in its opinions about the role of exercise in weight loss. Do we need more research to resolve this issue, or is it a question of analysis and debate? One dilemma is that the amount (dose) of exercise required to make a serious impact on body weight is perceived to be unrealistically high and therefore unachievable; on the other hand an amount of physical activity that would be tolerable (and even enjoyable) would hardly be effective. Also, there are issues concerning the accuracy of measurement of physical activity under free-living conditions. Questionnaires are not very precise, and movement sensors can be unreliable and relatively insensitive to some types of activity. Has research really informed us accurately enough about the amount of physical activity that people are actually performing? We can be sure that it is not enough. One step forward would be an improvement in the technology of instrumentation. Regarding wider environmental issues, the problem is the way in which the environment is structured to favour sedentariness. Reducing the amount of sedentary behaviour is recognized as different from increasing physical activity and possibly a more achievable objective. For children (and sometimes for adults) there is often a conflict between priorities; for example in the trade off between safety and adventure (risk). Environments perceived as dangerous are not conducive to the promotion of outdoor physical activity. Considering biological benefits, there is increasing interest in a developmental role for physical activity in improving mental functioning and strengthening the immune system. These benefits can occur separately from any effect on body weight. In this domain, the advantages of increasing physical activity and decreasing sedentariness are not in doubt; research is convinc- ing. However, research is needed into ways to empower people to engage in physical activity, and in constructing an environment (physical and socio-cultural) that enables people to make the healthy choices and prevents people from being lured into unhealthy behavioural patterns. The goal for current and future research is clear.

With the focus on the behavioural underpinnings of obesity - and especially paediatric obesity -, epidemiological research is strong although the sensitivity of measures used in large population studies can always be improved. Paediatric obesity is strongly heritable, but the current molecular genetic analyses cannot explain the heritability. Whereas the charting and recording of paediatric obesity is adequate (and has illustrated the scale of the problem), instigating behavioural change is extremely difficult. Concerning behavioural interventions, social cognitive models have been useful in describing the processes that lead to change, but far less effective in actually producing change. Deliberate (volitional) control over personal behaviour (eating and exercise) is very difficult to achieve for those who need it most (who are probably predisposed to gain weight). Research has taken us so far theoretically but has failed to deliver the mechanisms for implementing change. Is it realistic to expect this? Perhaps behaviour change requires the political will rather than more research? Can research deliver a behaviour breakthrough?

However, there is still a serious lack of understanding of the importance of behaviour (in comparison with genetics, biology, and nutrition) and how to manage it at the population level. There may also be some apprehension about failing to understand behaviour itself. At the individual level, clinical psychology has developed effective procedures to bring about changes in behaviour and associated cognitions.

To what extent has research on appetite and food intake behaviour really helped in understanding the problems of obese people? Research has demonstrated the complexity of processes controlling appetite and indicated that behaviour, and sensations, can be generated by the environment as well as by biology. Indeed the impact of the 'obesogenic' environment is mediated through explicit and implicit effects on cognitions and behaviour. Research has also identified behavioural components of the susceptible phenotype and has provided a taxonomy for discussing vulnerability to weight gain. However, articulating a description of this situation is not the same thing as building a management strategy (as has been noted for other domains of research). Indeed a certain amount of appetite-related research actually serves to support the food industry component of the obesogenic environment.

Taking a broad overview it can be perceived that scientific research has advanced markedly since the first European Congress on Obesity (ECO) in Stockholm in 1987 - almost a quarter of a century ago. There has been a proliferation of books, journals, congresses and symposia, scientific articles, and press releases of breakthroughs on a regular basis. Indeed 
there have been breakthroughs but what has been their impact on the understanding of the aetiology and management of obesity? During a 25-year period of immense research activity, the obesity epidemic has continued to grow, suggesting that research is not providing the information or the knowhow to suppress the causes of population weight gain. Indeed, it maybe questioned that, despite the accumulation of knowledge and facts, the cause of the 'epidemic' remain elusive. It has been proposed that the comforting energy balance model may not be capable of explaining secular changes in body weight during crucial periods of increase in obesity during the last 50 years. Is it possible then that we still do not really understand what 'causes' obesity? Perhaps research is a separate undertaking that can exist in parallel to an ongoing epidemic, and drawing justification (and funding) from the growing social and economic burden. What are the implications of this for planning our future research strategy?
Is this the time for a serious reconsideration of the value of obesity research? How much more knowledge is needed and in which areas? Should only translational research be funded? The SAB of EASO has begun a process of examination of the true value of research in our field of interest. This synopsis is only a partial reflection of what has been discussed. In turn, what has been discussed is not a complete representation of the entire spectrum of research. Many areas have not been covered, but deserve to be examined in the near future. It may take a short while to make a comprehensive review of all aspects of research, but it is the ambition of EASO to invigorate our thinking, to stimulate reflection, and to initiate a continuous dialogue with its members on these crucial issues. This process will be continued as part of EASO work programme relying on EASO SAB - and will be carried on during our next congresses in Istanbul (2011), Lyon (2012), and Liverpool (2013). 